\title{
The impact of atypical hyperplasia at the margins of breast-conserving surgery on the outcomes of breast cancer in patients treated with neoadjuvant chemotherapy
}

\author{
Heran Deng ${ }^{1}$, Jing Zhang ${ }^{1}$, Tingting $\mathrm{Hu}^{1}$, Qian $\mathrm{Li}^{1}$, Yaping Yang ${ }^{1}$, Jieqiong $\mathrm{Liu}^{1}$, and \\ Jiannan $\mathrm{Wu}^{1}$ \\ ${ }^{1}$ Sun Yat-Sen Memorial Hospital
}

August 4, 2021

\begin{abstract}
Purpose Women with atypical hyperplasia $(\mathrm{AH})$ is associated with a higher risk of later breast cancer. However, whether $\mathrm{AH}$ found at margins in patients with breast-conserving surgery (BCS) and neoadjuvant chemotherapy (NAC) needs re-excision is not well-defined. The aim of the present study was to evaluate the impact of atypical hyperplasia at the surgical margins on the local recurrence and survival outcomes in breast cancer patients treated with NAC and BCS. Methods A retrospective analysis comparing patients who received NAC with $\mathrm{AH}$ and received no re-excision to those without $\mathrm{AH}$ at the margins of BCS was performed. Results 323 patients were included in this study. The 5-year rates of ipsilateral breast tumor recurrence (IBTR) were $6 \%$ and $4.5 \%$ in patients with and without AH, respectively. Distant-metastasis-free survival (DMFS) at 5 years was $81.2 \%$ in the $\mathrm{AH}$ group, and $88.1 \%$ in the no-AH group. No significant differences were observed among the two groups in terms of IBTR, DMFS, or OS. Conclusion Our study suggests that AH involved at the surgical margins of BCS in patients who received NAC does not increase the risk of ipsilateral breast cancer, and there is insufficient evidence for surgeon to further resect $\mathrm{AH}$ found at the margins of $\mathrm{BCS}$ in these patients.
\end{abstract}

\section{Introduction}

Neoadjuvant chemotherapy (NAC) was originally one of the standard treatments for local advanced breast cancer. However, NAC is now increasingly being used among patients with early-stage breast cancer, because it can reduce the tumor size and convert patients who were initially candidates for mastectomy to be candidates for breast conserving surgery $(\mathrm{BCS})^{1,2}$ Additionally, NAC can eliminate metastatic disease in reginal lymph nodes which may change the surgical strategy of the axilla. It is established that a negative margin should be obtained when performing breast-conserving therapy. Compared with negative margins $(>2 \mathrm{~mm}$ ), positive margins (defined as ink on ductal carcinoma in situ or invasive carcinoma) lead to a twofold increase in the risk of local recurrence ${ }^{3}$. To date, several factors have been studied to be associated with the increased risk of local recurrence after breast-conserving surgery, including lymphovascular invasion, large tumor size, positive nodal status, extensive intradutal component, close or involved margin status, negative hormone receptor status ${ }^{4}$. Atypical hyperplasia $(\mathrm{AH})$ of the breast which can be categorized as atypical ductal hyperplasia (ADH) and atypical lobular hyperplasia (ALH), is a premalignant lesion, refers to abnormal epithelial proliferative breast lesions that are not qualitatively or quantitatively abnormal enough to be classified as carcinoma in situ ${ }^{5}$. It has been established that patients with AH on breast biopsy of benign lesions have an approximate four-fold increased risk of later breast cancer ${ }^{6,7}$. However, whether AH can also lead to increased local recurrence in patients underwent breast-conserving surgery remains unknown, especially in patients who have received NAC. Thus far, several studies have explored the issue of whether $\mathrm{AH}$ at margins of breast conserving surgery leads to increased local recurrence in the ipsilateral breast, and 
their conclusions were contradictory ${ }^{8-10}$. Lennington et al. found that ADH is often located at the periphery of ductal carcinoma in situ (DCIS) ${ }^{11}$, thus ADH identified at the margin of a BCS specimen may represent DCIS component is already very close to the tumor margin. And close margin may be related to higher local recurrence of the breast ${ }^{4}$. Therefore, it needs to be further investigated whether AH at margins is associated with local recurrence of breast cancer. To address this, our group previously reported a series of 244 breast cancer patients without NAC treated with BCS between 2009 and $2011^{12}$. We found that patients with $\mathrm{AH}$ at the margins experienced the same local control as those without AH. However, patients treated with NAC were excluded from our previous study. So far, there is no study to investigate the impact of atypical hyperplasia at margins on local recurrence and long-term survival outcomes in the NAC population. Therefore, the aim of the present study was to evaluate the impact of atypical hyperplasia at the surgical margins on the local recurrence and survival outcomes in breast cancer patients treated with NAC and BCS.

\section{Methods}

\section{Patients}

Institutional databases were reviewed to identify stage I-III breast cancer patients who received NAC, BCS at Sun Yat-sen Memorial Hospital, Sun Yat-sen University from 2011 to 2016. This study was approved by the Institutional Review Board (IRB) (SYSECKY-KS-2020-116)at Sun Yat-sen Memorial Hospital. All patients were proven to have invasive breast carcinoma by core needle biopsy. Clinicopathologic data including demographics, clinical oncologic features (tumor size, nodal stage) and tumor complete receptor information (ER, PgR, and HER2) was collected. The clinical and pathologic stages were defined according to the $8^{\text {th }}$ edition of the American Joint Committee on Cancer guidelines. Clinical stage was determined by ultrasound of the breast and lymph nodes. Lymph nodes with an abnormal appearance on ultrasound were routinely evaluated with a core-needle biopsy. A cutoff of $1 \%$ was used to determine the HR status on the core biopsy specimens. Tumors were defined as HER2 positive if they were $3+$ by immunohistochemistry or demonstrated gene amplification by fluorescence in situ hybridization ${ }^{13}$. Overall pathologic complete response (pCR) was defined as no residual invasive cancer in the breast or axillary lymph nodes.

\section{Treatment and pathologic considerations}

All of the patients received a complete course of neoadjuvant chemotherapy consisting of taxane, anthracycline, or both, and trastuzumab was given to patients with HER2+ breast cancer. After neoadjuvant chemotherapy, all patients underwent breast conserving surgery, axillary staging surgery with sentinel lymph node biopsy (SLNB) and/or axillary lymph node dissection (ALND), and irradiation therapy. When performing lumpectomy (BCS) after NAC, we remove the tumor with approximate $1 \mathrm{~cm}$ grossly negative margin. After completion of this lumpectomy, margins were evaluated by shaving the walls of the lumpectomy cavity, which was described previously by Chen $\mathrm{K} \mathrm{et} \mathrm{al}^{14}$. An intraoperatively frozen section analysis was performed to evaluate whether the tumor was involved in the cavity margins, and if the tumor was involved in the cavity margins, then additional excision of the involved margins for approximate $1 \mathrm{~cm}$ width (not the whole circle of the cavity wall) was performed until free margins were obtained, AH but no tumor at the margin was also defined as a negative margin. Postoperative paraffin-embedded hematoxylin and eosin (H\&E) staining was used to confirm the pathology diagnosis in the cavity margin specimens. AH at the margin was reviewed by two dedicated pathologists to verify the diagnosis in the tumor-free margin specimens. Additional adjuvant chemotherapy, targeted therapy (trastuzumab), and endocrine therapy were given when necessary according to the NCCN guidelines. There are two types of atypical hyperplasia, atypical ductal hyperplasia(ADH) and atypical lobular hyperplasia(ALH), which are based on their microscopic appearance, and ADH and ALH occur with equal frequency and confer a similar risk of breast cancer ${ }^{7}$. Therefore, in this study, we analyzed these two pathological features together as "atypical hyperplasia" (AH). ADH and ALH were diagnosed according to the criteria established by Page et $\mathrm{al}^{15}$. In atypical ductal hyperplasia, the involved ducts are filled and distended by monotonous epithelial cells forming architecturally complex patterns, including cribriform-like secondary lumens or micropapillary formations. Atypical lobular hyperplasia is characterized by expanded lobular acini filled with small, monotonous, round or polygonal cells, including a lack of cohesion and a loss of acinar lumens ${ }^{7}$. 


\section{Statistical analysis}

The primary end point of the present study was ipsilateral breast tumor recurrence (IBTR), defined as recurrence in the ipsilateral breast. The time to IBTR was calculated from the date of breast conserving surgery to the occurrence of IBTR, and it was censored at the time of the last follow-up or the time of death among patients who did not suffer IBTR. Patients characteristics of the two groups were compared by means of the chi-square test and the Kruskal-Wallis test, where appropriate. Kaplan-Meier method was used to estimate IBTR-survival, distant-metastasis-free survival (DMFS), and overall survival (OS). Differences between groups were compared with the log-rank test. Multivariate analyses were carried out using the Cox proportional hazards model. All values were two sided, and statistical significance was defined as $P<0.05$. All calculations were performed with SPSS 19.0 software (SPSS Inc., IBM, Chicago, USA).

\section{Results}

We identified 323 patients in this retrospective study, among them, 138 patients had AH at the margins in BCS, while 185 patients did not have this pathological feature at the margins. The median follow-up was 48 months (range 13-117). The comparison of clinicopathological characteristics and neoadjuvant chemotherapy response rate between the two groups is presented in Table 1. Patients with AH or without AH did not differ significantly by age, tumor histologic type, initial clinic $\mathrm{T}$ stage, initial nodal stage, receptor status, post-NAC pathologic $\mathrm{T}$ stage, post-NAC pathologic nodal status or overall $\mathrm{pCR}$ rate.

During the follow-up period, $8(5.8 \%)$ patients in the $\mathrm{AH}$ group, and $8(4.3 \%)$ patients in the non-AH group experienced ipsilateral breast tumor recurrence (IBTR). The 5-year rates of IBTR were $6.7 \%(95 \%$ CI, $4.4 \% \sim 9.0 \%)$ and $4.8 \%(95 \%$ CI, 3.1\% 6.5\%) in patinets with and without AH, respectively. Distantmetastasis-free survival (DMFS) at 5 years was $86.3 \%$ (95\% CI, 82.2\% 90.4\%) in the AH group, and $89.8 \%\left(95 \%\right.$ CI, $\left.87.4 \%^{\sim} 92.2 \%\right)$ in the non-AH group, respectively. Additionally, the 5 -years overall survival (OS) rate of the patients with or without $\mathrm{AH}$ was $93.1 \%$ (95\% CI, 90.5\% 95.7\%) and 93.8\% (95\% CI, $91.7 \% \sim 95.9 \%$ ), respectively. No significant differences were observed between the two groups of patients in terms of IBTR, DMFS, or OS ( $p=0.523,0.461$ and 0.328 , respectively). Atypical hyperplasia can be further classified into mild, moderate, and severe categories which borders on ducal carcinoma in situ. Coopey et al . evaluated breast cancer events in a retrospective cohort of 2938 women with ADH, ALH, LCIS, and severe $\mathrm{ADH}$, and the 10 years risk of breast cancer they estimated was $17 \%$ for women with $\mathrm{ADH}, 26$ for women with severe $\mathrm{ADH}^{16}$. Therefore, severe atypical hyperplasia may be regarded as "higher level of risk" lesion. We further analyzed the outcomes between patients with severe atypical hyperplasia and those without atypical hyperplasia, and no significant differences were found in IBTR, DMFS, or OS between patients with severe atypical hyperplasia $(\mathrm{n}=44)$ and those without atypical hyperplasia $(\mathrm{n}=185)$.

An overall pCR of breast and axillary nodes was achieved in 68 patients. Patients who achieved an overall pCR had significantly better DMFS $(p=0.022)$ and OS $(p=0.016)$, but not IBTR $(p=0.365)$, compared with those with residual disease. Among 255 patients with residual disease after neoadjuvant chemotherapy, 37 patients received re-excision due to invasive cancer and/or in situ carcinoma at the primary margins, 63 patients received re-excision due to severe $\mathrm{AH}$ which is somewhat difficult to distinguish from low grade DCIS in the frozen section ${ }^{17}$. Among these 63 patients, 16 still had severe AH after re-resection, and 3 of these 16 patients experienced local recurrence, while 1 of 30 patients without $\mathrm{AH}$ at re-excision margins had local recurrence, but again no significantly difference between the two group of these 46 patients in term of local recurrence in ipsilateral breast $(p=0.059)$.

It has been reported previously that some clinical, pathologic, and molecular factors were associated with IBTR after $\mathrm{BCS}^{4}$. Therefore, a multivariate analysis was performed to assess these factors associated with IBTR, DMFS and OS in our study. There was no association between atypical hyperplasia status and IBTR in the multivariate analysis. Similarly, other clinical and pathological features, including age, tumor histologic type, initial clinic $\mathrm{T}$ stage, initial nodal stage, receptor status, post-NAC pathologic $\mathrm{T}$ stage, post-NAC pathologic nodal status or overall pCR rate, were not significantly associated with IBTR as well. On multivariate analysis, patients who achieved pCR $(p=0.037$, HR 4.6, 95\% CI 1.09-19.18) had better 
DMFS, and patients who had negative lymph nodes ( $p=0.012$, HR 4.8, 95\% CI 1.41-16.79) after NAC had better OS.

\section{Discussion}

In this study, we extended our previous conclusions to patients who received NAC and BCS. Atypical hyperplasia at the margins also did not confer worse local ipsilateral breast recurrence-free survival, DMFS or OS in patients underwent BCS after NAC, and the observed IBTR rate was low (16/323, 5.0\%).

Data from a Netherlands study showed higher rates of tumor-involved margins in patients treated with NAC and BCS versus primary BCS (23 vs. $10 \%)^{2}$. Consistent with this literature, we found a high re-excision rate $30.9 \%(100 / 323)$ in our study. The rate of tumor at margins requiring intraoperative re-excision was actually quite low (37 patients, 11.4\%). Although 63 patients received intraoperative re-excision due to severe AH appeared at primary resection margins, the local recurrence rate of patients with severe AH was not higher than the patients without AH. In addition, Coopey et al . reported that patients with ADH and severe ADH were equally likely to develop invasive cancer and DCIS ${ }^{16}$. This indicates that if intraoperative frozen pathology can be accurately distinguish severe AH from DCIS, then there is no need to further resect severe $\mathrm{AH}$ at the margins. Our findings therefore lend further support for the oncology safety of residual severe $\mathrm{AH}$ at the margins to minimize unnecessary re-excisions.

$\mathrm{AH}$ of the breast refers to spectrum of abnormal epithelial proliferative lesions that does not qualify as carcinoma in situ. AH is found in approximately $10 \%$ of breast biopsies with benign lesions, and it is classified as a high-risk precancerous lesion due to its association with, and potential to progress to breast cancer $^{18}$. Women with $\mathrm{AH}$ have an approximate four-fold increase in $\mathrm{BC}$ risk ${ }^{6,7}$. Why $\mathrm{AH}$ did not confer higher IBTR in the present study, the following reasons may address to this issue. First, the conclusion that AH contributes to higher risk of breast cancer was drawn from people with benign diseases but not patients who have already suffered from breast cancer. The tumor burden of breast cancer patients was significantly different from those with only benign disease. Holland et al.reported in their study, of the 282 patients with invasive cancers that underwent lumpectomy, 105 (37\%) showed no other tumor foci in the residual breast, but other tumor foci were found in the remaining 177 breasts, of which $20 \%$ tumor foci were present within $2 \mathrm{~cm}$ off the reference tumor in the residual breast ${ }^{19}$. Therefore, it is clearly a negative margin that does not indicate that there is no residual cancer in the breast, however, the residual tumor can be controlled by radiotherapy and adjuvant treatments such as endocrine therapy and targeted therapy. The risk of IBTR of breast conserving therapy is about $0.5-2 \%$ per year with an increased risk during the first few years ${ }^{20,21}$, and the median time to IBTR is 36 months $^{22}$. Additionally, results from NSBAPB-18 suggested that patients downstaged after NAC for BCS may have higher local recurrence rate ${ }^{23}$. However, the annual incidence of breast cancer caused by AH is not significantly higher than the risk of IBTR after BCS. Recent reports with long-term follow-up have demonstrated that absolute risk for developing breast cancer is in the range of 1-2\% per year ${ }^{7,24}$. More recently, Menes and colleagues' research which was large and contemporary, found that the 10 years cumulative risk for developing breast cancer in women with atypical ductal hyperplasia (ADH) is only $5.6 \%{ }^{25}$. Therefore, the effect of AH on IBTR may be offset by the breast cancer itself. Thus, it is not surprising that $\mathrm{AH}$ involved at the margin does not contribute to a higher risk of IBTR. Second, endocrine therapy can reduce IBTR. Data from the NSABP P-1 trial showed that tamoxifen administered for 5 years decreased the risk of invasive and non-invasive breast cancer by approximately $50 \%{ }^{26}$. In the present study over $70 \%$ of the patients received endocrine therapy. We postulate that this result is attributable in part to the use of endocrine therapy. Third, the follow-up period of our study may not be long enough to reveal the effectiveness of the difference between groups. Page et al. reported that the interval of ADH and ALH to breast cancer is 8.2 and 11.9 years $^{27}$, but the median follow-up period of our study was approximately 48 months.

In addition to margin status, other important determinants of overall prognosis including the pCR rate, are being recognized. It has been reported that different chemotherapy responses and residual tumor burdens have different risks of cancer relapse, since patients who achieved a pCR will have favorable DFS and OS ${ }^{28,29}$. In the present study, patients who achieved a pCR had more excellent DMFS compared with those with 
residual lesions after NAC in our study. In patients undergoing NAC, the lack of an axillary pCR has been shown to be associated with less favorable overall survival ${ }^{30-32}$. Consistent with these published studies, we also found that positive lymph nodes post-NAC was associated with inferior OS.

Our study has some potential limitations due to its retrospective design. The chemotherapy regimens given to the patients varied among different subtypes, and this may affect the relationship between NAC response and DFS/OS. Furthermore, the number of patients enrolled in the study was limited. Future multi-centered studies and more patients enrolled with a longer follow-up are needed to achieve greater statistical power so as to address this issue more clearly.

In summary, our study did not find a higher risk of IBTR or distant metastasis or death events in patients with AH involved at the surgical margin who underwent BCS after NAC. In multivariate analysis, there was no association between AH status and IBTR, DMFS or OS. The lack of a pCR, and positive pathologic lymph nodes post-NAC were associated with inferior outcomes. Although further studies are needed to determine how AH status is related to IBTR in this population, the excellent long-term outcomes demonstrated in patients with $\mathrm{AH}$ at margins following NAC in this study suggest that $\mathrm{AH}$ involved at the margins may be acceptable in appropriately selected patients with breast cancer.

\section{Acknowledgements}

This work was supported by grants from the Natural Science Foundation of China (Grant 81702615/H1622).

Conflict of interest The authors have no conflict of interest to disclose.

Ethical Statement All procedures performed in this study involving human participants were in accordance with the ethical standards of the institutional and/or national research committee and with the 1964 Helsinki declaration and its later amendments or comparable ethical standards. Permission was gained from the local ethics committee of Sun Yat-sen Memorial Hospital for this retrospective analysis. For this type of study, formal consent was not required. All data were recorded and analysed anonymously.

\section{References}

1. Rastogi P, Anderson SJ, Bear HD, et al. Preoperative chemotherapy: updates of national surgical adjuvant breast and bowel project protocols B-18 and B-27. Journal of Clinical Oncology. 2008;26(5):778-785.

2. Volders J, Haloua M, Krekel N, et al. Neoadjuvant chemotherapy in breast-conserving surgeryConsequences on margin status and excision volumes: a nationwide pathology study.European Journal of Surgical Oncology (EJSO). 2016;42(7):986-993.

3. Moran MS, Schnitt SJ, Giuliano AE, et al. Society of Surgical Oncology-American Society for Radiation Oncology consensus guideline on margins for breast-conserving surgery with whole-breast irradiation in stages I and II invasive breast cancer. International Journal of Radiation Oncology* Biology* Physics.2014;88(3):553564.

4. Fragomeni SM, Sciallis A, Jeruss JS. Molecular Subtypes and Local-Regional Control of Breast Cancer.Surgical oncology clinics of North America. 2018;27(1):95-120.

5. Myers DJ, Walls AL. Atypical Breast Hyperplasia. In: StatPearls. Treasure Island (FL): StatPearls Publishing

Copyright (C) 2021, StatPearls Publishing LLC.; 2021.

6. Worsham MJ, Abrams J, Raju U, et al. Breast cancer incidence in a cohort of women with benign breast disease from a multiethnic, primary health care population. The breast journal. 2007;13(2):115-121.

7. Hartmann LC, Degnim AC, Santen RJ, Dupont WD, Ghosh K. Atypical hyperplasia of the breast-risk assessment and management options. New England Journal of Medicine.2015;372(1):78-89. 
8. Fowble B, Hanlon A, Patchefsky A, et al. The presence of proliferative breast disease with atypia does not significantly influence outcome in early-stage invasive breast cancer treated with conservative surgery and radiation. International Journal of Radiation Oncology* Biology* Physics. 1998;42(1):105-115.

9. Goldstein NS, Lacerna M, Vicini F. Cancerization of lobules and atypical ductal hyperplasia adjacent to ductal carcinoma in situ of the breast: significance for breast-conserving therapy. American journal of clinical pathology. 1998;110(3):357-367.

10. Arora S, Menes TS, Moung C, Nagi C, Bleiweiss I, Jaffer S. Atypical ductal hyperplasia at margin of breast biopsy —is re-excision indicated? Annals of surgical oncology. 2008;15(3):843-847.

11. Lennington WJ, Jensen RA, Dalton LW, Page DL. Ductal carcinoma in situ of the breast. Heterogeneity of individual lesions. Cancer. 1994;73(1):118-124.

12. Li S, Liu J, Yang Y, et al. Impact of atypical hyperplasia at margins of breast-conserving surgery on the recurrence of breast cancer. Journal of cancer research and clinical oncology. 2014;140(4):599-605.

13. Hammond MEH, Hayes DF, Dowsett M, et al. American Society of Clinical Oncology/College of American Pathologists guideline recommendations for immunohistochemical testing of estrogen and progesterone receptors in breast cancer (unabridged version). Archives of pathology $\& 3$ laboratory medicine.2010;134(7):e48e72.

14. Chen K, Zeng Y, Jia H, et al. Clinical outcomes of breast-conserving surgery in patients using a modified method for cavity margin assessment. Annals of surgical oncology. 2012;19(11):3386-3394.

15. Page DL, Rogers LW. Combined histologic and cytologic criteria for the diagnosis of mammary atypical ductal hyperplasia. Human pathology. 1992;23(10):1095-1097.

16. Coopey SB, Mazzola E, Buckley JM, et al. The role of chemoprevention in modifying the risk of breast cancer in women with atypical breast lesions. Breast Cancer Res Treat. 2012;136(3):627-633.

17. Tozbikian G, Brogi E, Vallejo CE, et al. Atypical ductal hyperplasia bordering on ductal carcinoma in situ: interobserver variability and outcomes in 105 cases. International journal of surgical pathology. 2017;25(2):100107.

18. Simpson JF. Update on atypical epithelial hyperplasia and ductal carcinoma in situ. Pathology.2009;41(1):36-39.

19. Holland R, Veling SH, Mravunac M, Hendriks JH. Histologic multifocality of tis, T1-2 breast carcinomas implications for clinical trials of breast-conserving surgery. Cancer. 1985;56(5):979-990.

20. Recht A, Silen W, Schnitt SJ, et al. Time-course of local recurrence following conservative surgery and radiotherapy for early stage breast cancer. International Journal of Radiation Oncology* Biology* Physics. $1988 ; 15(2): 255-261$.

21. Kurtz JM, Amalric R, Brandone H, et al. Local recurrence after breast-conserving surgery and radiotherapy. Frequency, time course, and prognosis. Cancer.1989;63(10):1912-1917.

22. DiPaola RS, Orel SG, Fowble BL. Ipsilateral breast tumor recurrence following conservative surgery and radiation therapy. Oncology (Williston Park, NY).1994;8(12):59-68; discussion 71, 75.

23. Swisher SK, Vila J, Tucker SL, et al. Locoregional control according to breast cancer subtype and response to neoadjuvant chemotherapy in breast cancer patients undergoing breast-conserving therapy. Annals of surgical oncology.2016;23(3):749-756.

24. Hartmann LC, Radisky DC, Frost MH, et al. Understanding the premalignant potential of atypical hyperplasia through its natural history: a longitudinal cohort study. Cancer Prevention Research. 2014;7(2):211217. 
25. Menes TS, Kerlikowske K, Lange J, Jaffer S, Rosenberg R, Miglioretti DL. Subsequent breast cancer risk following diagnosis of atypical ductal hyperplasia on needle biopsy. JAMA oncology. 2017;3(1):36-41.

26. Fisher B, Costantino JP, Wickerham DL, et al. Tamoxifen for the prevention of breast cancer: current status of the National Surgical Adjuvant Breast and Bowel Project P-1 study. Journal of the National Cancer Institute.2005;97(22):1652-1662.

27. Page DL, Dupont WD, Rogers LW, Rados MS. Atypical hyperplastic lesions of the female breast. A long-term follow-up study. cancer. 1985;55(11):2698-2708.

28. Dowsett M, Dunbier AK. Emerging biomarkers and new understanding of traditional markers in personalized therapy for breast cancer. Clinical Cancer Research.2008;14(24):8019-8026.

29. Cortazar P, Zhang L, Untch M, et al. Pathological complete response and long-term clinical benefit in breast cancer: the CTNeoBC pooled analysis. The Lancet.2014;384(9938):164-172.

30. Cho DH, Bae SY, You JY, et al. Lymph node ratio as an alternative to $\mathrm{pN}$ staging for predicting prognosis after neoadjuvant chemotherapy in breast cancer. The Kaohsiung journal of medical sciences. 2018;34(6):341-347.

31. van Nijnatten TJ, Simons J, Moossdorff M, et al. Prognosis of residual axillary disease after neoadjuvant chemotherapy in clinically node-positive breast cancer patients: isolated tumor cells and micrometastases carry a better prognosis than macrometastases. Breast cancer research and treatment. 2017;163(1):159-166.

32. Choi J, Laws A, Hu J, Barry W, Golshan M, King T. Margins in Breast-Conserving Surgery After Neoadjuvant Therapy. Ann Surg Oncol. 2018;25(12):3541-3547.

\section{Hosted file}

Table 1.docx available at https://authorea.com/users/429084/articles/532837-the-impact-ofatypical-hyperplasia-at-the-margins-of-breast-conserving-surgery-on-the-outcomes-ofbreast-cancer-in-patients-treated-with-neoadjuvant-chemotherapy 